\title{
Corrections to Weighted Opacities and Energy Exchange Rate in 3-T Radiation-Hydrodynamics
}

\author{
K. W. McLean and S. J. Rose \\ Plasma Group, Imperial College London, South Kensington, London, SW7 2AZ, UK
}

\begin{abstract}
It is often the case that high energy density systems can be well described and simulated in the 3T approximation, where electrons, ions and the radiation field are defined at unique temperatures given by $T_{e}, T_{i}, T_{r}$. The difference in temperature between the electrons and radiation field is important when calculating weighted opacities and electronradiation energy exchange rates. Often, it is assumed that $T_{r} \approx T_{e}$, meaning the quantities can be calculated as functions of $T_{e}$ only. This paper explores the consequences that arise when one uses this assumption in regions where $T_{r} \neq T_{e}$. Mutliplicative correction factors are derived for the Rosseland and Planckian mean opacities $\left(\kappa_{R}\right.$ and $\left.\kappa_{P}\right)$ and for the electron-radiation energy exchange rate. We find that there exists a very small region of parameter space where the corrections are negligible. However, for the majority of parameter space explored, numerical corrections vary from factors of 2 to multiple orders of magnitude.
\end{abstract}

Keywords: HED, 3-T radiation hydrodynamics, Opacity, Diffusion, Rosseland mean, Planckian mean

\section{Introduction}

Three-temperature (3T) models of plasmas in which the electrons, ions and radiation are treated locally and described by separate temperatures $\left(T_{e}, T_{i}\right.$ and $T_{r}$, respectively) have been used in plasma modelling for many years [1], [2], [3], [4]. For ease of computation the approximation is often made in the evaluation that the opacities and electron-radiation exchange rate are functions of electron temperature only, whereas in reality both factors depend on the electron and radiation temperatures. In this paper, we derive and evaluate multiplicative correction factors to the Planckian and Rosseland mean opacities and the electron-photon energy exchange rates to correct for this. Results for each correction factor are given for Iron $(Z=26)$ and Aluminium $(\mathrm{Z}=13)$.

\section{Theory}

The equation of radiative transfer describes how energy contained within a propagating beam of light changes along its trajectory due to interactions with surrounding material and is given by

$$
\frac{1}{c} \frac{\partial I_{v}}{\partial t}+\boldsymbol{n} \cdot \nabla I_{v}=\rho \kappa_{v}\left[B_{v}\left(T_{e}\right)-I_{v}\left(T_{r}\right)\right]
$$

where $I_{v}$ is the specific intensity, $\rho$ is the mass density, $\kappa_{v}$ is the opacity and $B_{v}$ denotes the blackbody function. The unit vector $\mathbf{n}$ is directed along the ray-path The derivation of equation 1 is given in many different forms across the literature, such as in [5], [6] and [7]. Note that any opacities used in this work are assumed to be already corrected for stimulated emission. Scattering effects are also ignored.

Consecutive multiplication of equation 1 by $\mathbf{n}$ and integration over solid angle, $\mathrm{d} \Omega$, allows the derivation of a set of moment equations. The zeroth moment describes how the energy contained within the beam changes as the radiation is absorbed and emitted. This is given by

$$
\frac{\partial E_{v}}{\partial t}+\nabla \cdot \boldsymbol{F}_{v}=\rho \kappa_{v}\left[4 \pi B_{v}\left(T_{e}\right)-E_{v} c\right]
$$

Where $E_{v}$ is the energy density and $F_{v}$ is the radiative flux, which are both frequency dependent quantities. The first order moment defines the radiation momentum equation as

$$
\frac{1}{c} \frac{\partial \boldsymbol{F}_{v}}{\partial t}+c \nabla \cdot \boldsymbol{P}_{v}=-\rho \kappa \boldsymbol{F}_{v}
$$

Where $\mathbf{P}$ is the pressure tensor. To close this set of equations, and solve for $E_{v}$, it is necessary to add another, artificial, constraint to the system. The most com- 
mon solution is to assume that the system is in the radiative diffusive regime, where the material is sufficiently optically thick that radiation diffuses down the radiative temperature gradient through repeated absorption and emission. In this regime the photon mean free path, $\lambda$, satisfies

$$
\lambda=[\rho \kappa]^{-1} \ll L
$$

Where $L$ is the characteristic length-scale over which the macroscopic properties of the plasma change. Also, we assume that the specific intensity $I_{v}$ is close to isotropic and can be expressed as

$$
I_{v} \approx I_{0}+\mu I_{1}
$$

Where $\mu \ll 1$ and and $I_{0}$ is isotropic. Describing the radiation field in this way implies that the pressure tensor can be expressed explicitly in terms of the energy density

$$
\boldsymbol{P} \rightarrow P=\frac{E}{3}
$$

This, along with equations 4 and 3, can be used to derive a form of Fick's law [8] which relates the radiative flux to energy density, given by

$$
\frac{c}{3} \nabla E_{v}=-\rho \kappa_{v} \boldsymbol{F}_{v}
$$

Substituting into 2 gives the radiation energy equation in the diffusive regime as

$$
\frac{\partial E_{v}}{\partial t}=\nabla \cdot D_{v} \nabla E_{v}+4 \pi \rho \kappa_{v}\left[B_{v}\left(T_{e}\right)-B_{v}\left(T_{r}\right)\right]
$$

Where the energy density is related to the blackbody function by

$$
E_{v}(T)=\frac{4 \pi}{c} B_{v}(T)
$$

and the diffusion coefficient is given by

$$
D_{v}=\frac{c}{3 \rho \kappa_{v}}
$$

To solve for total energy density, $E$, equation 8 is integrated across the entire frequency spectrum. In doing this, one obtains

$$
\frac{\partial E}{\partial t}=\nabla \cdot \frac{c}{3 \rho \kappa_{R}} \nabla E+\rho a c\left[\kappa_{P}\left(T_{e}\right) T_{e}^{4}-\kappa_{P}\left(T_{e}, T_{r}\right) T_{r}^{4}\right]
$$

Where the Rosseland mean, $\kappa_{R}$, and Planckian mean, $\kappa_{P}$, are defined as follows

$$
\kappa_{R}^{-1}\left(\rho, T_{e}, T_{r}\right)=\frac{\int \kappa^{-1}\left(\rho, v, T_{e}\right) \frac{\partial B\left(v, T_{r}\right)}{\partial T_{r}} d v}{\int \frac{\partial B\left(v, T_{r}\right)}{\partial T_{r}} d v}
$$

$$
\kappa_{P}\left(\rho, T_{e}, T_{r}\right)=\frac{\int_{0}^{\infty} \kappa\left(\rho, v, T_{e}\right) B\left(v, T_{r}\right) d v}{\int_{0}^{\infty} B\left(v, T_{r}\right) d v} \ldots
$$

Note that both these functions are dependent on two temperatures, with $\kappa_{P}\left(T_{e}\right)$ denoting the specific case of the weighting function and the opacity being dependent on the same temperature, $T_{e}$.

The Rosseland and Planckian mean are often referred to as the transport and absorption-emission opacities respectively. This is due to their role in the equation of radiation transport; $\kappa_{R}$ appears in the term accounting for the diffusion of radiation down a temperature gradient, whereas $\kappa_{P}$ appears in the term accounting for the enhancement and degredation of the radiation intensity due to interactions with matter. One further quantity explored in this paper is the energy exchange rate between electrons and photons, denoted by $\omega^{e r}$. The exchange rate describes how much energy is given to the material via absorption, minus how much is removed via emission and is given by the right hand side of equation 11 .

$$
\omega^{e r}=\rho a c\left[\kappa_{P}\left(T_{e}\right) T_{e}^{4}-\kappa_{P}\left(T_{e}, T_{r}\right) T_{r}^{4}\right]
$$

It may be the case that only single temperature weighted opacities are available to use in rad-hydro codes - particularly in those which use table-fed opacities. The following sections will investigate the consequences of these assumptions through derivation and evaluation of a multiplicative correction factor for $\kappa_{P}, \kappa_{R}$ and $\omega^{e r}$. The form of each correction factor is identical; the ratio of the correct, two temperature, result to that obtained where one assumes $T_{r}=T_{e}$.

\section{Free-free Opacities}

In the case where opacity is dominated by freefree contributions (such as in a fully ionised plasma), Kramers's approximation ([6], [9]) is often used:

$$
\rho \kappa_{v}^{f f}=\frac{4 e^{6}}{3 m h c}\left(\frac{2 \pi}{3 k m}\right)^{\frac{1}{2}} T_{e}^{-\frac{1}{2}} Z^{2} n_{e} n_{i} g_{f f} v^{-3}\left(1-e^{-\frac{h \nu}{k T_{e}}}\right)
$$

which makes it possible to analytically integrate both 12 and 13. In cases for which this does not apply and where bound-free and bound-bound processes are important we use opacities calculated from the Ionised Material Package (IMP) code [10]. 


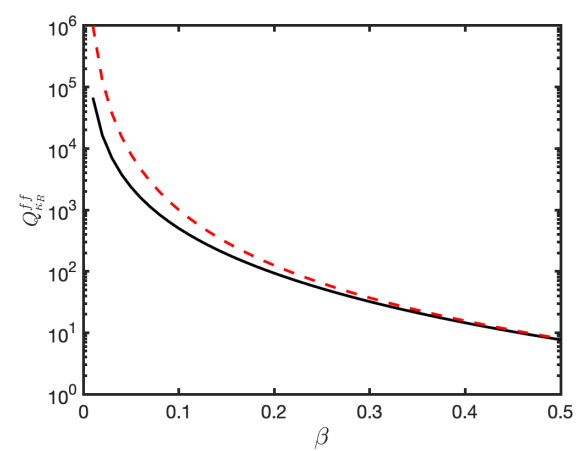

Figure 1: Correction factor for Rosseland mean opacity assuming the opacity is dominated by purely free-free interactions. The correction factor is shown with a solid black line, with the red dashed line representing a $\beta^{-3}$ decline which $Q_{K_{R}}^{f f}$ tends to as $\beta$ increases. Above $\beta=0.5$ both forms are almost identical.

\subsection{Rosseland Mean Opacity}

The correction factor for the Rosseland mean opacity, denoted by $Q_{\kappa_{R}}$, is given by

$$
Q_{\kappa_{R}}=\frac{\kappa_{R}\left(T_{e}, T_{R}\right)}{\kappa_{R}\left(T_{e}\right)}
$$

This can be expressed analytically when the opacity is given by 15 , where $Q_{\kappa_{R}}$ is given by

$$
Q_{\kappa_{R}}^{f f}=\beta^{-3} \cdot \frac{I_{1}}{I_{2}}
$$

where

$$
\begin{gathered}
I_{1}=\int \frac{x^{7} e^{x}}{\left(e^{x}-1\right)^{2}} \frac{d x}{\left(1-e^{-\beta x}\right)} \\
I_{2}=\int \frac{x^{7} e^{2 x} d x}{\left(e^{x}-1\right)^{3}} \\
\beta=\frac{T_{R}}{T_{e}} \\
x=\frac{h v}{k_{B} T_{R}}
\end{gathered}
$$

As $\beta$ increases, $I_{1} \rightarrow I_{2}$ and the correction factor takes on the form of an inverse cubic function of the temperature ratio. This is shown in figure 1 .

\subsection{Planckian Opacity and Energy Exchange Rate}

Results are presented for the correction factors to the Planckian opacity and the energy exchange rate obtained for $\kappa_{P}$ and how they affect $\omega^{e r}$. It is necessary to explain how the two correction factors are related. $Q_{\kappa_{P}}$, is given by

$$
Q_{\kappa_{P}}=\frac{\kappa_{P}\left(T_{e}, T_{r}\right)}{\kappa_{P}\left(T_{e}\right)}
$$

The correction factor to the energy exchange rate is subtly different, it corrects the value of $\omega^{e r}$ where one has assumed that $\kappa_{P}\left(T_{e}, T_{r}\right) \approx \kappa_{P}\left(T_{e}\right)$. Using this assumption, the correction factor to the exchange rate, $Q_{\omega^{e r}}$, is

$$
Q_{\omega^{e r}}=\frac{1-Q_{\kappa_{P}} \beta^{4}}{1-\beta^{4}}
$$

where $\beta$ has the same definition as in 17 .

For the case where the opacity is given by $15, Q_{K_{P}}$ is very similar to that given in 17. $Q_{\omega^{r}}^{f f}$ is given by

$$
Q_{\omega^{e r}}^{f f}=\beta \cdot \frac{(-\psi(\beta)-\gamma)}{1-\beta^{4}}
$$

where $\psi$ is the digamma function and $\gamma$ is the Euler constant [11] defined by

$$
\int_{0}^{\infty}\left(\frac{e^{-t}-e^{-\beta t}}{1-e^{-t}}\right) d t=\psi(\beta)+\gamma
$$

The digamma function is plotted in figure 2 .

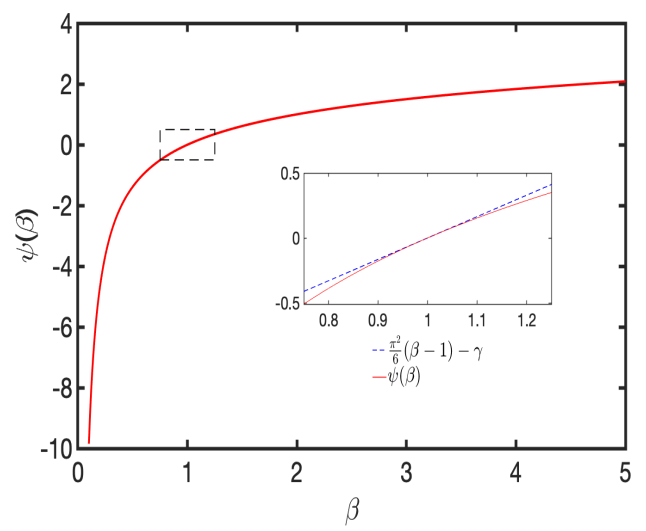

Figure 2: Form of digamma function, $\psi$, for $0<\beta<5$. The immediate neighbourhood around $\beta \approx 1$ is magnified to demonstrate the accuracy of the further assumption given in equation 22 .

Around $\beta \approx 1$, the digamma function can be further expanded

$$
\psi(\beta)+\gamma \approx \frac{\pi^{2}}{6}(\beta-1)
$$

This is shown as the region bounded by the dashed rectangle in figure 2 , which is also shown magnified to show the exact range of $\beta$ values across which this approximation is reasonable. In this region, the electronradiation exchange rate can be approximated as: 


$$
\omega^{e r}=\Lambda \frac{T_{r}}{T_{e}}\left(T_{e}-T_{r}\right)
$$

where

$$
\Lambda=\frac{16 e^{6}}{9 m h c^{3}}\left(\frac{2 \pi^{7} k}{3 m}\right)^{\frac{1}{2}} T_{e}^{-\frac{1}{2}} Z^{2} n_{e} n_{i} g_{f f}
$$

The general form of $Q_{\omega^{r}}^{f f}$, analytically solved using properties of $\psi$ is shown in figure 3. At $\beta=1$, equation 20 becomes undefined as $\psi(1)=-\gamma$. To solve at this point L'Hôpital's rule was used and yielded a value of $Q_{\omega^{e r}}^{f f}(\beta=1) \approx 0.411$. The same problem arises in the numerical case and manifests itself as sudden breaks in the line plots obtained for $Q_{\omega^{e r}}$ in section 3 .

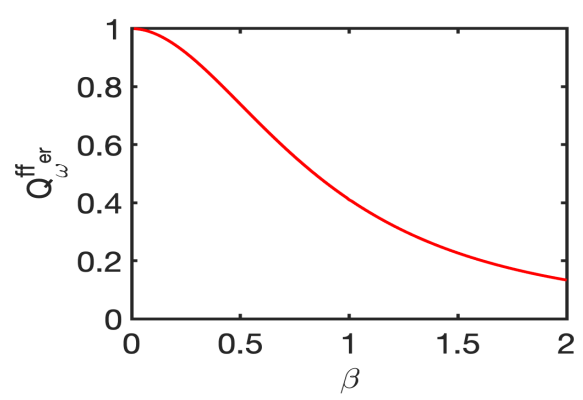

Figure 3: Analytical correction factor of electron-radiation energy exchange rate, $Q_{\omega^{e r}}^{f f}$, calculated using properties of the digamma function, $\psi$.

\section{General Opacities}

When bb and bf components of opacity are included, equations 16,18 and 19 must be solved numerically using opacity profiles provided by IMP [10], which calculates radiative opacity profiles in plasmas including contributions from bound-bound and bound-free interactions. Results are simulated for the same conditions as those in section 3 and are listed in figures 4 through 6.
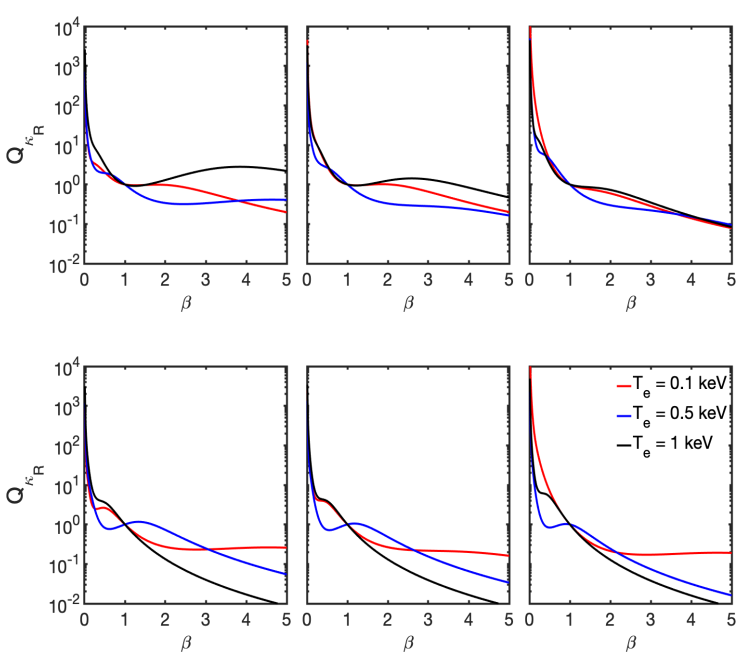

Figure 4: Values of the Rosseland mean opacity correction factor, $Q_{\kappa_{R}}$, shown for Iron (top) and Aluminium (bottom). From left to right the results are given at $\frac{1}{10} \rho_{s}, \rho_{s}$ and $10 \rho_{s}$ where $\rho_{s}$ is solid density; $7.87 \mathrm{gcc}$ for Iron and $2.7 \mathrm{gcc}$ for Aluminium.
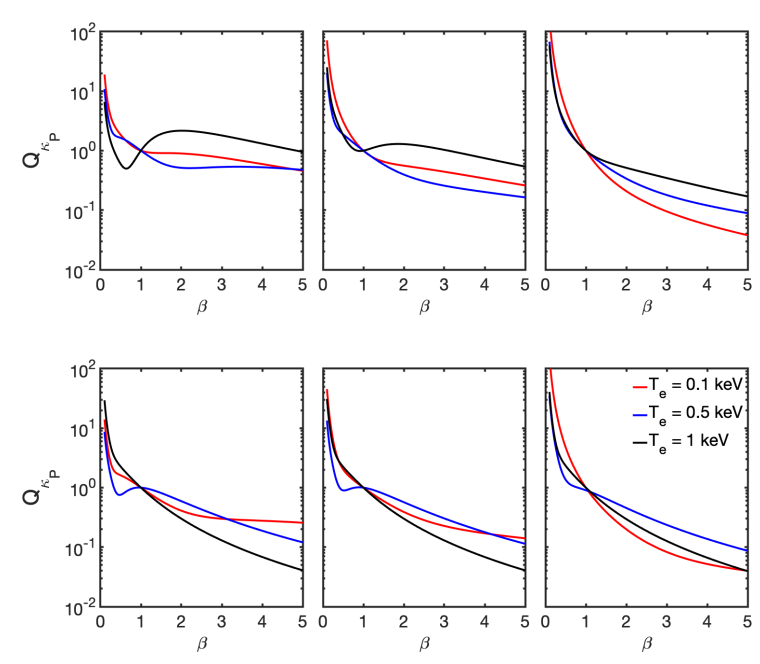

Figure 5: Values of the Planckian mean opacity correction factor, $Q_{\kappa P}$, shown for Iron (top) and Aluminium (bottom). From left to right the results are given at $\frac{1}{10} \rho_{s}, \rho_{s}$ and $10 \rho_{s}$ where $\rho_{s}$ is solid density; $7.87 \mathrm{gcc}$ for Iron and $2.7 \mathrm{gcc}$ for Aluminium. 

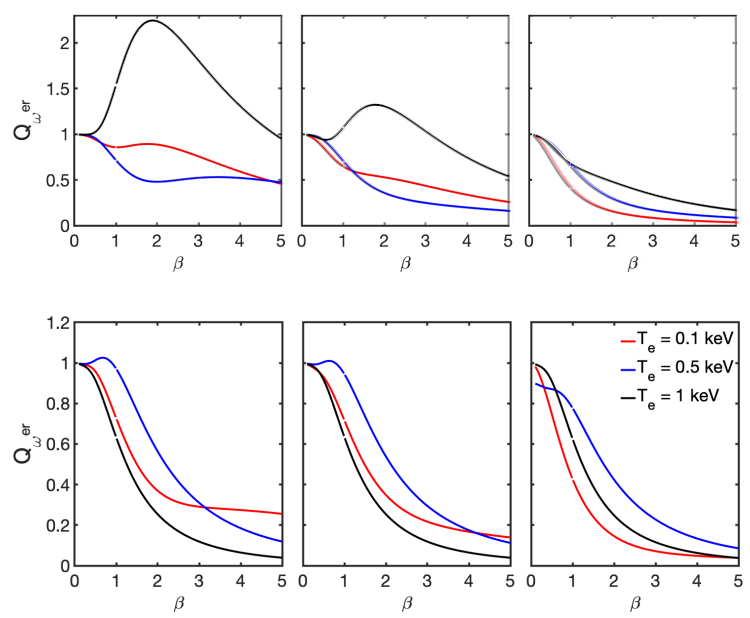

Figure 6: Values of the electron-radiation energy exchange correction factor, $Q_{\omega^{e r}}$, shown for Iron (top) and Aluminium (bottom). From left to right the results are given at $\frac{1}{10} \rho_{s}, \rho_{s}$ and $10 \rho_{s}$ where $\rho_{s}$ is solid density; $7.87 \mathrm{gcc}$ for Iron and $2.7 \mathrm{gcc}$ for Aluminium.

\section{Discussion}

The results presented show that in regimes where $T_{r} \neq T_{e}$, the correction factors deviate significantly from unity and depend on material, radiation temperature, $T_{r}$, electron temperature, $T_{e}$, and mass density, $\rho$.

\subsection{Rosseland Mean}

The results obtained clearly indicate the two temperature dependence of $\kappa_{R}$. There are particular regions where the correction factor becomes several orders of magnitude. In 3-T documentation [1], the user is advised that, in the case that opacities are given for one temperature only, the following approximation is should be used

$$
\kappa_{R}\left(T_{r}, T_{e}\right) \approx \kappa_{R}\left(T_{e}\right)
$$

It is clear from figure 4 that this approximation holds only in a small neighbourhood around the point of $T_{r} \approx T_{e}$. However, corrections are necessary for even small deviations from this. In some cases, particularly for Iron, a peak is present. The position of this peak depends on the frequency at which the opacity profile exhibits absorption lines and edges, which is why they are not present in the purely free-free analytical case. To explain this, consider the case that a region containing absorption lines or an ionisation edge overlaps with the peak of $\frac{\partial B}{\partial T}$. In this scenario, the integral of $\int \kappa_{v}^{-1} \frac{\partial B}{\partial T} d v$ becomes large and in turn causes $Q_{K_{P}}$ to increase accordingly, as is demonstrated in figure 7. At high temperatures and densities, the numerical and analytical solutions tend common values. This is expected in these conditions due to the almost complete ionisation leading to a free-free dominated opacity.

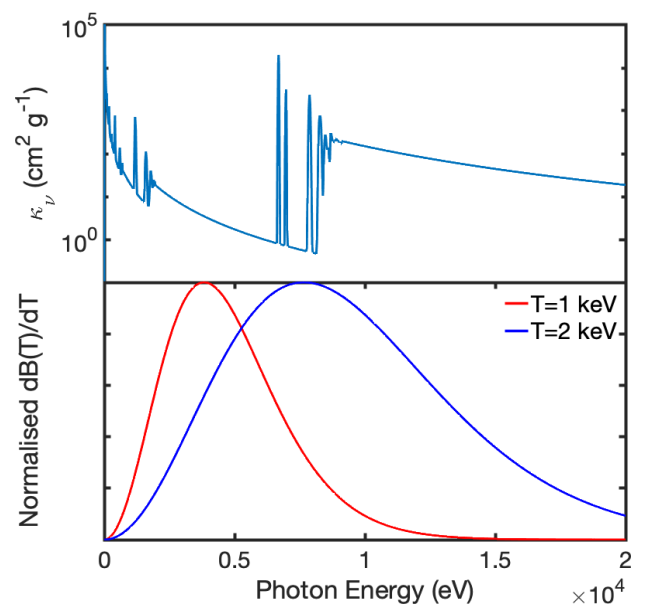

Figure 7: Plot of Iron opacity profile at $0.787 \mathrm{gcc}$ and $T_{e}=1 \mathrm{keV}$ (top). Normalised blackbody functions at temperatures of $1 \mathrm{keV}$ (blue) and $2 \mathrm{keV}$ (red) (bottom). The correction factor $Q_{k_{R}}$ for the iron conditions stated is maximised at $\beta \approx 2$ due to the frequency of absorption lines matching up with the peak of $\frac{d B}{d T}$.

\subsection{Planckian Mean and Energy-Exchange Rate}

The results obtained for $\kappa_{P}$ and $Q_{\omega^{e r}}$ follow many similar trends to those discussed above. The same peak formation appears in the form of $Q_{\kappa p}$, again due to the overlapping of the opacity profile with the relevant weighting function. The only difference in this case is that the weighting function is the blackbody function itself rather than the temperature derivative. Again, the peak occurs at around $\beta=2$; the frequency difference between the peaks of $\frac{d B(T)}{d T}$ and $B(T)$ is very small at these temperatures.

The plots of $Q_{\omega^{e r}}$ show that the corrections necessary for the exchange rate are less severe than the other two quantities. For the majority of conditions explored, not accounting for radiation-electron temperature differences causes an over-evaluation of $\omega^{e r} ; Q_{\omega^{e r}}<1$. However under-evaluation occurs within regions in parameter space where the blackbody fuction peak, now evaluated at the radiation temperature, overlaps lines and edges in the opacity profile. The region where $Q_{\omega^{e r}} \approx 1$ corresponds to $T_{r} \rightarrow 0$, which agrees with the first order approximation advised in 3-T documentation [1]

$$
\omega^{e r}\left(T_{e}, T_{r}, \rho\right) \approx \omega^{e r}\left(T_{e}, 0, \rho\right)
$$



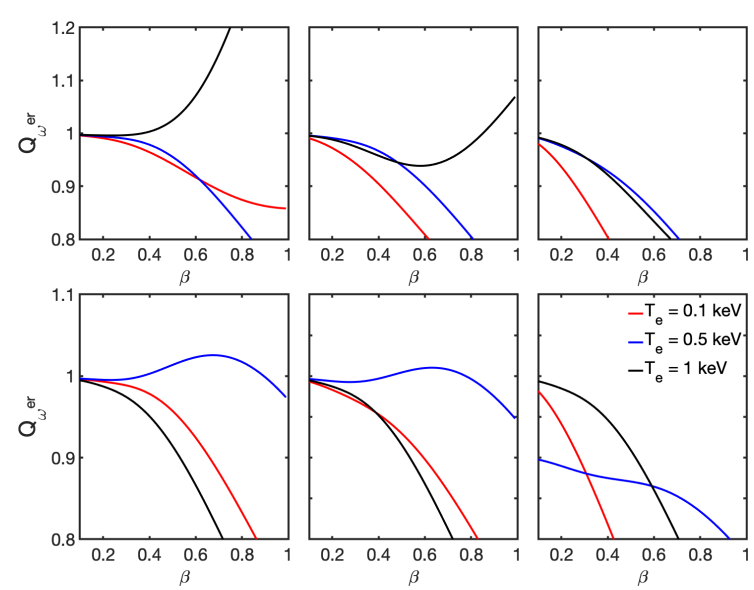

Figure 8: Identical plots to those given in figure 6 magnified in the region $0<\beta<1$, corresponding to $0<T_{r}<T_{e}$.

A magnified plot of the exchange rate between the points of $T_{r}=0$ and $T_{r}=T_{e}$ is given in figure 8 .

The approximation given in equation 26 becomes inaccurate around $\beta \approx 0.5$ and, outside this region, leads to corrections which can be up to a factor 2 .

At high temperatures and densities, the numerical values of both $Q_{\kappa_{P}}$ and $Q_{\omega^{e r}}$ tend to the analytical cases highlighting that in particular regimes, the digamma function is all that needs to be evaluated to correct these parameters.

\section{Conclusions and Future Work}

The work presented highlights the derivation and evaluation of correction factors aimed to account for differences in electron temperature, $T_{e}$, and radiation temperature, $T_{r}$, when calculating the Rosseland mean opacity, Planckian mean opacity and electron-radiation energy exchange rate. The results indicate that there are very small regions of parameter space where the difference in temperature results in a negligible correction and in many cases, corrections are necessary. The extent of these corrections depends on the plasma density, $\rho$, material $Z$ as well as electron and radiation temperatures. The purpose of this study was not to provide multiplicative corrections for all cases but to provide indicative values for specific materials.

The factors derived in this study could be implemented as a full revision of existing table fed opacities, which is a common method of feeding opacity values into radiation-hydrodynamics simulations. Alternatively, separate tables of correction factors could be stored for each value of $\beta$ and used to modify any existing opacity tables where necessary. The f-f dominated regime would be the most simple to implement, due to the correction factors taking analytical form. However, one should take care in using this approximation as it will only be valid when the system is clearly dominated by f-f interactions (very close to completely ionised). In all other cases, where b-f and b-b interactions are important, there will be no scaling laws and the correction will have to be solved numerically for each specific set of conditions.

Although this work has focused primarily on 3-T radiation algorithms, the same problem would arise in under-resolved multigroup radiation solvers. If any weighted opacity is used with the incorrect weighting function, then inaccuracies will arise. If the frequency space is resolved sufficiently, then $\kappa_{P} \approx \kappa_{R} \rightarrow \kappa$, and the weighting function becomes irrelevant; it is under these circumstances that the correction factors become obsolete.

[1] M. M. Basko, Nucl. Fusion 302443.

[2] B. Fryxell, K. Olson, P. Ricker, F. X. Timmes, M. Zingale, D. Q. Lamb, P. Macneice, R. Rosner, J. W. Truran, H. Tufo, et al., Flash: An adaptive mesh hydrodynamics code for modeling astrophysical thermonuclear flashes, The Astrophysical Journal Supplement Series 131 (1) (2000) 273334. doi:10.1086/317361.

[3] G. Fraley, E. Linnebur, R. Mason, R. Morse, Thermonuclear burn characteristics of compressed deuterium-tritium microspheres, Physics of Fluids 17 (2). doi:10.2172/4429729.

[4] N. A. Tahir, K. A. Long, E. W. Laing, Method of solution of a three-temperature plasma model and its application to inertial confinement fusion target design studies, Journal of Applied Physics 60 (3) (1986) 898-903. doi:10.1063/1.337330.

[5] G. C. Pomraning, The equations of radiation hydrodynamics, Dover Publications, 2005.

[6] G. B. Rybicki, A. P. Lightman, Radiative processes in astrophysics, Wiley, 2004.

[7] J. I. Castor, Radiation hydrodynamics, Cambridge University Press, 2004.

[8] A. Fick, On liquid diffusion, Journal of Membrane Science 100 (1995) 3338.

[9] W. F. Huebner, W. D. Barfield, Opacity, Springer, 2014.

[10] S. J. Rose, Calculations of the radiative opacity of laserproduced plasmas, Journal of Physics B: Atomic, Molecular and Optical Physics 25 (7) (1992) 1667-1681. doi:10.1088/09534075/25/7/034.

[11] M. Abramowitz, I. A. Stegun, Handbook of mathematical functions, Dover, 1972. 